\title{
A Remote Multi-touch Experience to Support Collaboration between Remote Museum Visitors
}

\author{
Ernesto Arroyo, Valeria Righi, Roger Tarrago, and Josep Blat \\ GTI, ICT Department, Universitat Pompeu Fabra, \\ Roc Boronat 138, 08018 Barcelona, Spain \\ \{ernesto.arroyo, valeria.righi, roger.tarrago, josep.blat\}@upf .edu
}

\begin{abstract}
This paper presents a collaborative experience designed to support learning in two remotely located museums sharing a common exhibition. A remote collaborative multi-touch experience offers an additional channel for museum visitors to explore the exhibition and increase the sense of connectedness and awareness between the two spaces. The experience flow includes stages offering opportunities for exploration, negotiation and cooperation. The paper describes the design and implementation of a system that allows simultaneous collaborative interaction and communication through two multi-touch surfaces augmented with videoconferencing. The system allows museum visitors to communicate with remote participants and with their peers. Finally, the paper discusses preliminary observations of end-users, and cultural organizations using the prototype. This work provides a use case for social interactive experiences that could draw museum visitors to further explore an exhibition and share their views and interpretation with others.
\end{abstract}

Keywords: Computer supported collaborative learning, multi-user interaction, informal learning, serious games, remote awareness, interactive surfaces.

\section{Introduction}

This project is framed within the context of two Spanish museums that exhibit an art collection simultaneously in Figueres and Barcelona cities. Each museum exhibits part of a collection with the intention to allow visitors to freely explore and experience the collection. These conditions offer interaction designers a unique opportunity to explore different ways for inter-connecting museums and its visitors using a common exhibition to build the discourse. This paper presents the design and implementation of a prototype designed to support collaboration and learning in remote museum environments. The prototype facilitates simultaneous collaborative interaction and communication through two multi-touch surfaces and a videoconferencing system; similar to the DigiTable project [7].

\subsection{Related Work}

Several museum guides, tools, and games have been developed to support locationaware collaboration, cooperation and interaction using devices that could be located 
anywhere within a museum [3][4]. However, few have been specifically designed for inter-connecting two remote museum spaces, e.g. [2]. On the other hand, online collaborative systems include videoconferencing and digital sharing capabilities that are already used in educational and cultural settings [10][9]. Additionally, tabletops have also been used to facilitate face-to-face collaborative learning. These tools allow simultaneous collaboration between multiple remote participants, which facilitate the provision of learning [5], support online collaboration activities [6] [7] and improve the cognitive proximity and identity decoupling of participants. Our work incorporates several technologies to make explicit the relationship between two "partner" museums hosting a common exhibition through a system composed of two stations placed in two remote locations.

\section{Remote Multi-touch Experience}

Together, with museum stakeholders, we designed a system targeted to students groups visiting a museum during a coordinated activity in two distant cities. The system seeks to offer an additional channel for museum visitors to explore the exhibition and increase the sense of connectedness between the two spaces by allowing museum visitors to interact and collaborate. Each station includes two 32' screens: one horizontal multi-touch screen, and a vertical screen (Fig. 1). The horizontal screen incorporates a multi-touch table that allows for direct manipulation of up to 32 objects and displays content related to the exhibition that can be seen and accessed simultaneously from both stations. The vertical screen displays a video feed from each location and complementary information related to the learning experience.

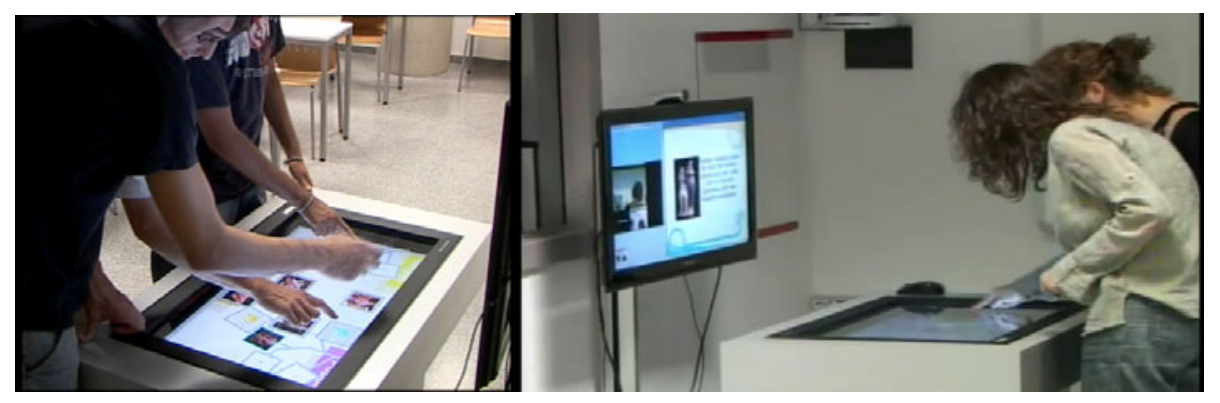

Fig. 1. The system includes two remotely located stations. Participants interact on a horizontal surface while sharing the same workspace with their remote partners. Left) co-located participants interacting simultaneously. Right) a vertical screen shows a video feed of the remote location facilitating communication between the two locations.

\subsection{Experience Description}

Groups of participants are invited to play a "game" with other groups also visiting a museum at a different city. The game is related to the objects presented in both museums. During the game, groups of participants interact with each other as each group holds key information related to the exhibition in their own cities that will help 
the other group complete the game tasks. The experience flow is modeled in three stages that offer different opportunities for exploration, negotiation and cooperation and that guide and facilitate discovering and exploring the installation materials. The first stage provides useful information for completing the other stages and it promotes negotiation by requiring participants to coordinate the material to be explored. The other two stages include focused collaborative tasks [1] that can be solved by collaborating with other participants and encourage information sharing between visitors. The system supports simultaneous manipulation over a shared collaborative workspace for users located remotely and users co-located. Remote users share the same visual perspective using a what you see is what I see interaction metaphor [8]. Several visual cues help participants understand the user actions in the remote museum. Finally, the system includes a videoconference service to promote communication and reflection between remote groups [1]. The aim is that museum visitors will use the videoconference to explain, and justify their actions over the virtual shared space.

\section{Preliminary Evaluation}

The experience went through an iterative design process where high school and university students informally tested several prototype versions. The experience has also been presented to several cultural and governmental organizations in two public events. The game lasted less than 10 minutes on average and we observed a learning curve regarding simultaneous interactions; on the first stage participants exhibited a turn-taking behavior, while on the second stage they interacted with the objects simultaneously and independently. We also observed a similar curve in regard to remote collaboration, which decreased as the game advanced. The videoconferencing proved useful as participants used it in a natural way to communicate with remote participants. Visual cues used to facilitate remote awareness proved useful in supporting simultaneous remote interaction; however, some participants claimed that the workspace seemed chaotic when all participants were moving items. Finally, we also found that individuals wanted to experience the installation by themselves without having a remote partner on the other end. This can be easily addressed by offering single and collaborative modes of interaction in future iterations.

\section{Conclusion and Future Work}

We have presented an informal learning experience designed to connect two remotely located museums sharing a common installation on. Our system allows museum visitors to communicate between and with their peers around a multi-touch tabletop through videoconferencing. We have validated that the system supports natural interaction and allows multiple visitors to interact simultaneously on a tangible shared space while also facilitating face-to-face collaboration among participants within the same location, and among remote participants. This project illustrates one possible 
way for inter-connecting museums and its visitors using a common exhibition. Future work includes evaluating several usability and interaction aspects of the system, identifying collaborative tasks suitable for this type of settings, and improving reliability issues required for everyday use.

Acknowledgments. This work has been partially funded by the Learn 3 project, (TIN2008-05163/TSI). The authors would like to thank the cultural and technical partners of the C3I2 workgroup for their support and ideas.

\section{References}

1. Baker, M., Lund, K.: Promoting reflective interactions in a CSCL environment. Journal of Computer Assisted Learning 13(3) (2003)

2. Galani, A., Chalmers, M.: Empowering the remote visitor: Supporting social museum experiences among local and remote visitors. In: 2nd International Conference of Museology (ICM), Mytiline, Greece (2004)

3. Ghiani, G., Paternò, F., Santoro, C., Spano, L.D.: UbiCicero: A location-aware, multidevice museum guide. Interacting with Computers 21(4), 288-303 (2009)

4. Laurillau, Y., Paternó, F.: Supporting Museum Co-visits Using Mobile Devices. In: Brewster, S., Dunlop, M.D. (eds.) Mobile HCI 2004. LNCS, vol. 3160, pp. 451-455. Springer, Heidelberg (2004)

5. Looi, C.-K., Chen, W., And Ng, F.-K.: Collaborative activities enabled by GroupScribbles (GS): An exploratory study of learning effectiveness. Computers \& Education 54(1), 14 26 (2010)

6. Palloff, R.M., Pratt, K.: Collaborating online. Learning together in community. JosseyBas, San Francisco (2005)

7. Pauchet, A., Coldefy, F., Lefebvre, L., et al.: Tabletops: Worthwhile Experience of Collocated and Remote Collaboration. In: Proc. TABLETOP 2007, pp. 27-34 (2007)

8. Stefik, M., Bobrow, D.G., Foster, G., Lanning, S., Tatar, D.: WYSIWIS revised: early experiences with multi-users interfaces. ACM Trans. Inf. Syst. 5(2), 147-167 (1987)

9. Vom Lehn, D., Heath, C., Hindmarsh, J.: Exhibiting Interaction: Conduct and Collaboration in Museums and Galleries. Symbolic Interaction Journal 24(2), 189-216 (2001)

10. Wunar, B., Kowrach, N.: Live...from the Heart: Using Interactive Videoconferencing to Link Classrooms to Museum Resources. In: Gibson, I., et al. (eds.) Society for IT \& Teacher Education International Conference, pp. 581-582 (2009) 